\title{
On the interpretation of the latest AMS-02 cosmic ray electron spectrum
}

\author{
Fiorenza Donato, ${ }^{a, b, *}$ Mattia Di Mauro ${ }^{b}$ and Silvia Manconi ${ }^{c}$ \\ ${ }^{a}$ Department of Physics, University of Torino, via P. Giuria, 1, 10125 Torino, Italy \\ ${ }^{b}$ Istituto Nazionale di Fisica Nucleare, via P. Giuria, 1, 10125 Torino, Italy \\ ${ }^{c}$ Institute for Theoretical Particle Physics and Cosmology, RWTH Aachen University, \\ Sommerfeldstr. 16, 52056 Aachen, Germany \\ E-mail: donato@to.infn.it
}

The latest AMS-02 data on cosmic ray electrons show a break in the energy spectrum around $40 \mathrm{GeV}$, with a change in the slope of about 0.1 . We perform a combined fit to the newest AMS02 positron and electron flux data above $10 \mathrm{GeV}$ using a semi-analytical diffusion model where sources includes production of pairs from pulsar wind nebulae (PWNe), electrons from supernova remnants (SNRs) and both species from spallation of hadronic cosmic rays with interstellar medium atoms. We demonstrate that within our setup the change of slope in the AMS-02 electron data is well explained by the interplay between the flux contributions from SNRs and from PWNe. In fact, the relative contribution to the data of these two populations changes by a factor of about 13 from 10 to $1000 \mathrm{GeV}$. The PWN contribution has a significance of at least $4 \sigma$, depending on the model used for the propagation, interstellar radiation field and energy losses. We checked the stability of this result against low-energy effects by solving numerically the transport equation, as well as adding possible breaks in the injection spectrum of SNRs. The effect of the energy losses alone, when the inverse Compton scattering is properly computed within a fully numerical treatment of the Klein-Nishina cross section, cannot explain the break in the $e^{-}$flux data, as recently proposed in the literature.

$37^{\text {th }}$ International Cosmic Ray Conference (ICRC 2021)

July 12 th - 23rd, 2021

Online - Berlin, Germany

\footnotetext{
${ }^{*}$ Presenter
} 


\section{Introduction}

In this paper we seek an interpretation of the latest $e^{ \pm}$data, and in particular to assess if the break in the $e^{-}$AMS-02 data at about $40 \mathrm{GeV}$ is explained by an energy loss effect as found in Refs. [1, 2], or by the interplay between the emission of different source populations, similarly to what found in [3] by fitting the $e^{+}+e^{-}$Fermi-LAT data. We adopt the density of the local interstellare radiation field (ISRF) in [5] ${ }^{1}$ (Vernetto2016) and test ISRF models of Refs. [2, 6, 7], use whether a smooth spatial Galactic distribution of PWNe and SNRs or by including Galactic spiral arms as in [10], and employ the propagation parameters in Refs. [1, 11]. We calculate, for the first time, the significance for the contribution of PWNe to the measured AMS-02 $e^{-}$flux and we statistically assess the nature of the break in the data. For all the details about modeling, implementations, and analysis results we refer to [4].

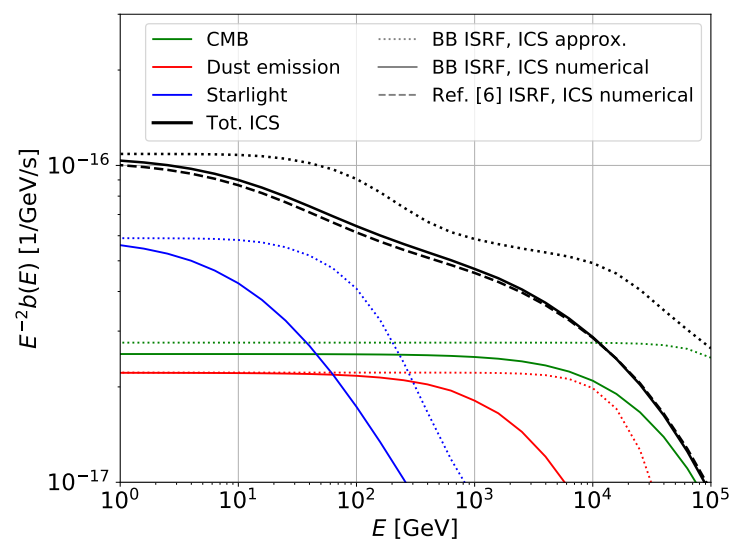

Figure 1: Energy loss rate $b(E)=d E / d t$ (multiplied by $E^{2}$ ) for ICS off the ISRF photons composed by CMB (green lines), dust emission (red lines) and starlight (blue lines), for $e^{ \pm}$energy $E$. The total rate is shown with black lines. We report three cases: black body approximations of the ISRF and approximated Klein-Nishina calculation as in [8] (dotted lines), black body approximations of the ISRF and full numerical Klein-Nishina calculation (solid lines), [5] ISRF model and full numerical Klein-Nishina calculation (no approximations, dashed line).

\section{Results}

We consider energy losses associated to synchrotron emission on the Galactic magnetic field, and ICS losses, which are demonstrated to dominate over other energy loss mechanism for $e^{ \pm}$ observed at Earth with $E>10 \mathrm{GeV}$. As a first result, we compute the energy loss term $b(E)=d E / d t$ as due to inverse Compton scattering (ICS). We consider a full numerical treatment of this process, as well as the analytic reduction taken from Ref. [8], used by [1] and observed to be a poor approximation by [9]. In Fig. 1 we show the energy loss term $b(E)$ as a function of $e^{ \pm}$energy $E$. It is clearly visible that the ICS approximation cases are significantly different from the Klein-Nishina exact calculation ones for each photon field, in particular when the Thomson regime does not apply.

\footnotetext{
${ }^{1}$ This reference appears as [6] in the labels of the figure.
} 


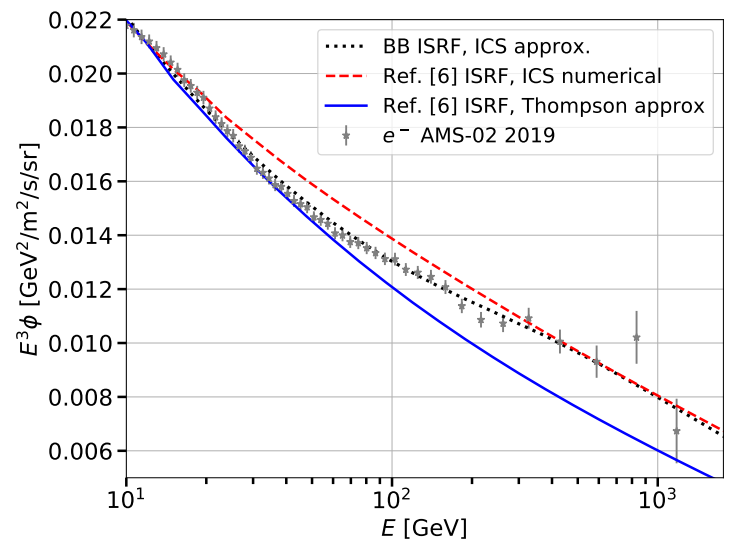

Figure 2: Flux of $e^{-}$from a smooth distribution of SNRs calculated for $\gamma_{\mathrm{SNR}}=2.55$. We show the same cases for the ISRF and the Klein-Nishina energy loss rate as the ones reported in Fig. 1, in order to demonstrate the effect of the approximated calculation of the ICS energy losses published in Ref. [8] and implemented in Ref. [1] on the $e^{-}$flux.
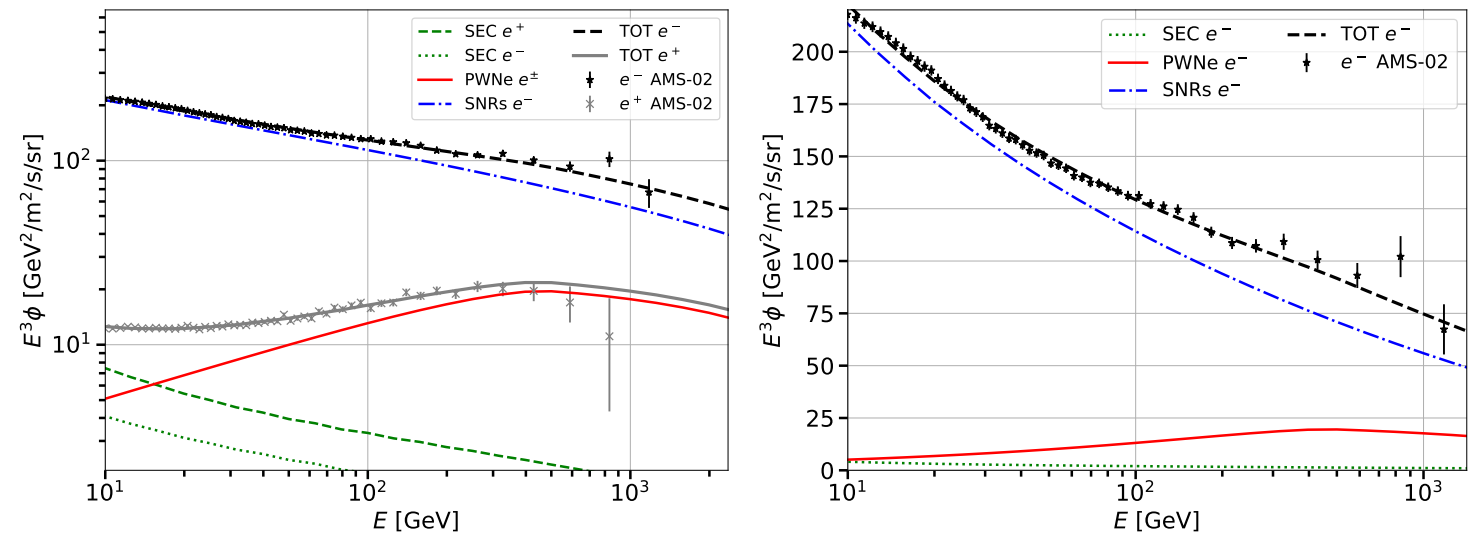

Figure 3: Left Panel: Result for the combined fit to $e^{-}$and $e^{+}$AMS-02 data (black and grey data points). We show the secondary production of $e^{+}$(dashed green line) and $e^{-}$(dotted green line), $e^{ \pm}$from PWNe (solid red line), $e^{-}$from SNRs (dot-dashed blue line). Right Panel: same as the left panel but zooming in the $e^{-}$ sector.

We implement the $b(E)$ cases reported in Fig. 1 with the Klein-Nishina loss rate (ICS numerical) and the approximated treatment (ICS approx). The $e^{-}$flux computed using the Thomson approximation is also reported for comparison. We fix $\gamma_{\mathrm{SNR}}=2.5$ and the Vernet to 2016 ISRF density. The result on the $e^{-}$spectrum is shown in Fig. 2, along with AMS-02 data. The flux predicted with the ICS numerical cases does not show any evident change of shape over the whole energy range, thus suggesting to exclude the option that the break in the $e^{-}$AMS-02 data might be due to the ICS energy losses.

We then perform a combined fit to the $e^{+}$and $e^{-}$AMS-02 data above $10 \mathrm{GeV}$ leaving free to vary the normalization of the secondary component $q$, the spectral index $\gamma_{\mathrm{SNR}}$, the SNR average energy per source $W_{\mathrm{SNR}}$, the efficiency $\eta_{\mathrm{PWN}}$ for the conversion of PWN spin-down luminosity into $e^{ \pm}$, and the spectral indexes $\gamma_{1}$ and $\gamma_{2}$ below and above the break for the PWN injection spectrum. 


\begin{tabular}{|c|c|c|c|c|c|c|c|c|c|c|c|}
\hline$\%$ & ISRF & Propagation & Spiral Arms & ICS & $q$ & $\gamma_{S N R}$ & $W_{\text {SNR }}$ & $\gamma_{1,2}$ & $\eta_{\text {PWN }}$ & $\tilde{\chi}^{2}$ & $\sigma_{P W N}$ \\
\hline & & & & & & {$\left[10^{49} \mathrm{erg}\right]$} & & & & \\
\hline 1 & Vernetto2016 & Genolini2015 & No & numerical & 1.32 & 2.57 & 1.35 & $1.88 / 2.31$ & 0.009 & 0.92 & 5.8 \\
2 & Vernetto2016 & Genolini2015 & Yes & numerical & 1.54 & 2.43 & 1.53 & $1.61 / 2.20$ & 0.017 & 1.64 & 8.2 \\
3 & Vernetto2016 & BPLDiffusion & No & numerical & 1.32 & 2.50 & 1.15 & $1.80 / 2.58$ & 0.010 & 0.82 & 4.0 \\
4 & Delahaye2010 & Genolini2015 & No & numerical & 1.31 & 2.59 & 1.44 & $1.90 / 2.27$ & 0.009 & 0.95 & 6.1 \\
5 & Delahaye2010 & BPLDiffusion & Yes & approx & 1.78 & 2.43 & 2.13 & $1.56 / 2.80$ & 0.018 & 0.71 & 0.2 \\
6 & Evoli10/2020 & BPLDiffusion & Yes & numerical & 1.50 & 2.56 & 3.34 & $1.82 / 2.21$ & 0.022 & 0.84 & 3.9 \\
\hline \hline 7 & Evoli10/2020 & Genolini2015 & No & numerical & 1.31 & 2.66 & 2.17 & $1.98 / 2.39$ & 0.011 & 0.89 & 4.0 \\
8 & Porter2006 & Genolini2015 & No & numerical & 1.35 & 2.58 & 1.24 & $1.87 / 2.23$ & 0.008 & 1.00 & 6.6 \\
9 & Vernetto2016 & Genolini2015 & No & approx & 1.41 & 2.56 & 1.38 & $1.84 / 2.80$ & 0.008 & 0.71 & 1.1 \\
10 & Evoli10/2020 & BPLDiffusion & No & numerical & 1.42 & 2.60 & 1.95 & $1.88 / 2.65$ & 0.011 & 0.78 & 6.4 \\
\hline \hline
\end{tabular}

Table 1: Summary of the results obtained with the combined fit to $e^{+}$and $e^{-}$AMS-02 data (see text for details). We show cases where we vary the ISRF model, diffusion parameters, where we include or not the Galactic spiral arms, use the numerical calculation of the Klein-Nishina ICS energy losses or employ the approximation in [8]. We list the best-fit values for the secondary renormalization factor $q$, SNR spectral index $\gamma_{S N R}$ and average energy emitted per source $W_{\text {SNR }}$, PWN source spectral indexes $\gamma_{1}$ and $\gamma_{2}$ below and above the break energy, the PWN efficiency $\eta_{\mathrm{PWN}}$ and the value of the best fit reduced chi-square $\tilde{\chi}^{2}$. The last column reports the significance for the PWN contribution.

\begin{tabular}{|c|c|c|c|c|c|c|c|}
\hline ISRF & Propagation & Spiral Arms & ICS & PWN & $\gamma_{S N R}$ & $W_{\text {SNR }}$ & $\chi^{2}$ \\
\hline & & & & & & {$\left[10^{49} \mathrm{erg}\right]$} & \\
\hline Vernetto2016 & Genolini2015 & No & Thomson & No & 2.47 & 0.94 & 142 \\
Vernetto2016 & Genolini2015 & No & numerical & No & 2.53 & 1.18 & 130 \\
Vernetto2016 & Genolini2015 & No & numerical & Yes & 2.57 & 1.35 & 89 \\
\hline Evoli102020 & BPLDiffusion & No & Thomson & No & 2.39 & 0.85 & 137 \\
Evoli102020 & BPLDiffusion & No & numerical & No & 2.54 & 1.60 & 125 \\
Evoli102020 & BPLDiffusion & No & numerical & Yes & 2.60 & 1.95 & 76 \\
\hline \hline
\end{tabular}

Table 2: Best-fit parameters of SNRs obtained through a fit to $e^{ \pm}$AMS-02 data. We report, for each model, in the first (second) row the case for which we calculate the energy losses in the Thomson approximation (Klein-Nishina, numerical) without accounting for the PWNe contribution to the $e^{-}$flux. The third row is for the losses calculated with the Klein-Nishina loss rate and adding also the PWNe $e^{-}$flux. The last column represents the $\chi^{2}$ obtained with the combined fit to $e^{ \pm}$AMS-02 data with 97 degrees of freedom.

We select data above $10 \mathrm{GeV}$ to minimize the effect of the solar modulation that, if not properly taken into account, could generate a bias in the results. We thus have 6 free parameters in the fit $\left(q, \gamma_{\mathrm{SNR}}, W_{\mathrm{SNR}}, \eta_{\mathrm{PWN}}, \gamma_{1}\right.$ and $\left.\gamma_{2}\right)$, and 103 data points. The fit is performed simultaneously to $e^{+}$ and $e^{-}$data. We show the results of the fit in Fig. 3 along with the AMS-02 data. We find a good agreement with the high-energy part of the $e^{+}$data with $\gamma_{1}=1.88$ and $\gamma_{2}=2.31$. We need an efficiency of about $\eta_{\mathrm{PWN}}=0.91 \%$ that is similar to the value required to explain the $\gamma$-ray halos detected in Fermi-LAT and HAWC data around the powerful Geminga and Monogem pulsars [12]. The model reproduces well both the $e^{+}$and $e^{-}$data in the entire energy range considered. Indeed, the reduced $\chi^{2}$ is equal to 0.93 .

We show the results in Tab. 2 for our benchmark model as well as using the Evoli10/2020 
ISRF and the BPLDiffusion propagation setup which is similar to the model used in Ref. [2]. We find for both models a relatively small improvement in the goodness of fit between the case of Thomson and the Klein-Nishina ICS losses, the increase in the $\chi^{2}$ being 12. Instead, the $\chi^{2}$ improves significantly, by a value of 41 and 37, when adding the PWN flux in the two models tested in Tab. 2. The fit on $e^{-}$data points, that are 52, improves significantly when adding PWNe into the model, while it changes mildly when calculating the losses with the Klein-Nishina formalism with respect to the Thompson approximation.

In Fig. 4 we show the result on the $e^{-}$flux at Earth for the first six cases tested in this Section and summarized in Tab. 1. When we only modify the ISRF model or propagation parameters with respect to the benchmark model, we obtain very similar contributions from the SNR and PWN fluxes. Instead, if we use the ICS energy losses approximation as in Ref. [8], and then implemented in Ref. [1], we find a change of trend in the SNR flux at around $100 \mathrm{GeV}$, similar to what found in Ref. [1]. In particular, since the SNR flux for this model shows an hardening with increasing energy, the PWN contribution is forced to be slightly lower than in the other cases, and the resulting fit is better. However, as demonstrated in [4], this model is based on a poor approximated calculation of the ICS energy losses, which we have shown to poorly reproduce the transition between the Thomson regime and Klein-Nishina formalism. The benchmark model, in which energy losses are computed using a fully numerical approach, fits very well the data.

\section{Conclusions}

We have demonstrated that the AMS-02 $e^{-}$and $e^{+}$flux data can be properly explained with the production of CR leptons from SNRs, PWNe and secondary production. Specifically, $e^{+}$above $10 \mathrm{GeV}$ are mostly explained with PWNe with a power-law injection spectrum broken at about $500 \mathrm{GeV}$, and a change of slope below and above the break of about $\Delta \gamma=0.5$. SNRs explain most of the $e^{-}$flux. Their contribution decreases with energy from $96 \%$ at $10 \mathrm{GeV}$ to $78 \%$ at 500 $\mathrm{GeV}$, while PWNe provide an increasing contribution reaching a maximal $21 \%$ at $500 \mathrm{GeV}$. For the first time, we estimated the significance of the PWN contribution to the $e^{-}$flux, that varies within $4-8 \sigma$, considering different models for the ISRF, source distribution in the Galaxy and propagation parameters. We also provided a statistical test to probe the hypothesis that the break at $40 \mathrm{GeV}$ detected in AMS-02 $e^{-}$data is due to the transition of ICS energy losses between the Thomson regime and the Klein-Nishina formalism on the starlight component. We quantitatively assess that the improvement in the fit by using the Klein-Nishina loss rate with respect to the Thomson approximation is much smaller than the one obtained with the addition of the PWNe flux in the model. The stability of our results against low-energy effects, such as convection and reacceleration, is checked by solving numerically the transport equation, as well as adding possible breaks in the injection spectrum of SNRs [4]. We thus conclude that the break measured by AMS-02 in the $e^{-}$ cosmic flux at $E \sim 40 \mathrm{GeV}$ is very likely due to the interplay between the contribution of SNRs and PWNe. 


\section{References}

[1] C. Evoli, P. Blasi, E. Amato, R. Aloisio Signature of Energy Losses on the Cosmic Ray Electron Spectrum Phys. Rev. Lett. 125 (2020) 051101

[2] C. Evoli, P. Blasi, E. Amato, R. Aloisio Galactic factories of cosmic-ray electrons and positrons Phys. Rev. D 103 (2021) 3010

[3] M. Di Mauro et al. Theoretical interpretation of Pass 8 Fermi-LAT $e^{+}+e^{-}$data Astrophys. J. 845 (2017) 107

[4] M. Di Mauro, F. Donato, S. Manconi On the interpretation of the latest AMS-02 cosmic ray electron spectrum subm. to Phys. Rev. D, eprint arXiv:2010.13825

[5] S. Vernetto and P. Lipari, Absorption of very high energy gamma rays in the Milky Way Phys. Rev. D 94 (2016) 3009

[6] T.A. Porter, I.V. Moskalenko and A.W. Strong Inverse Compton Emission from Galactic Supernova Remnants: Effect of the Interstellar Radiation Field Astrophys. J. 648 (2006) L29

[7] T. Delahaye et al., Galactic electrons and positrons at the Earth: new estimate of the primary and secondary fluxes Astron. \& Astrophys. 524 (2010) A51

[8] R. Schlickeiser and J. Ruppel Klein-Nishina steps in the energy spectrum of galactic cosmic-ray electrons New J. of Physics 12 (2010) 33044

[9] K. Fang, X-J. Bi and S-J. Lin, Klein-Nishina effect and the cosmic ray electron spectrum Chin. Phys. Lett. 38 (2021) 039801, arxiv:2007.15601

[10] R.J. Wainscoat et al., A Model of the 8-25 Micron Point Source Infrared Sky Astrophys. J. Suppl. S. 83 (1992) 111

[11] Y.Genolini et al., Theoretical uncertainties in extracting cosmic-ray diffusion parameters: the boron-to-carbon ratio Astron. \& Astrophys. 580 (2015) A9

[12] M. Di Mauro, S. Manconi, F. Donato Detection of a $\gamma$-ray halo around Geminga with the Fermi -LAT data and implications for the positron flux Phys. Rev. D 100 (2019) 123015 

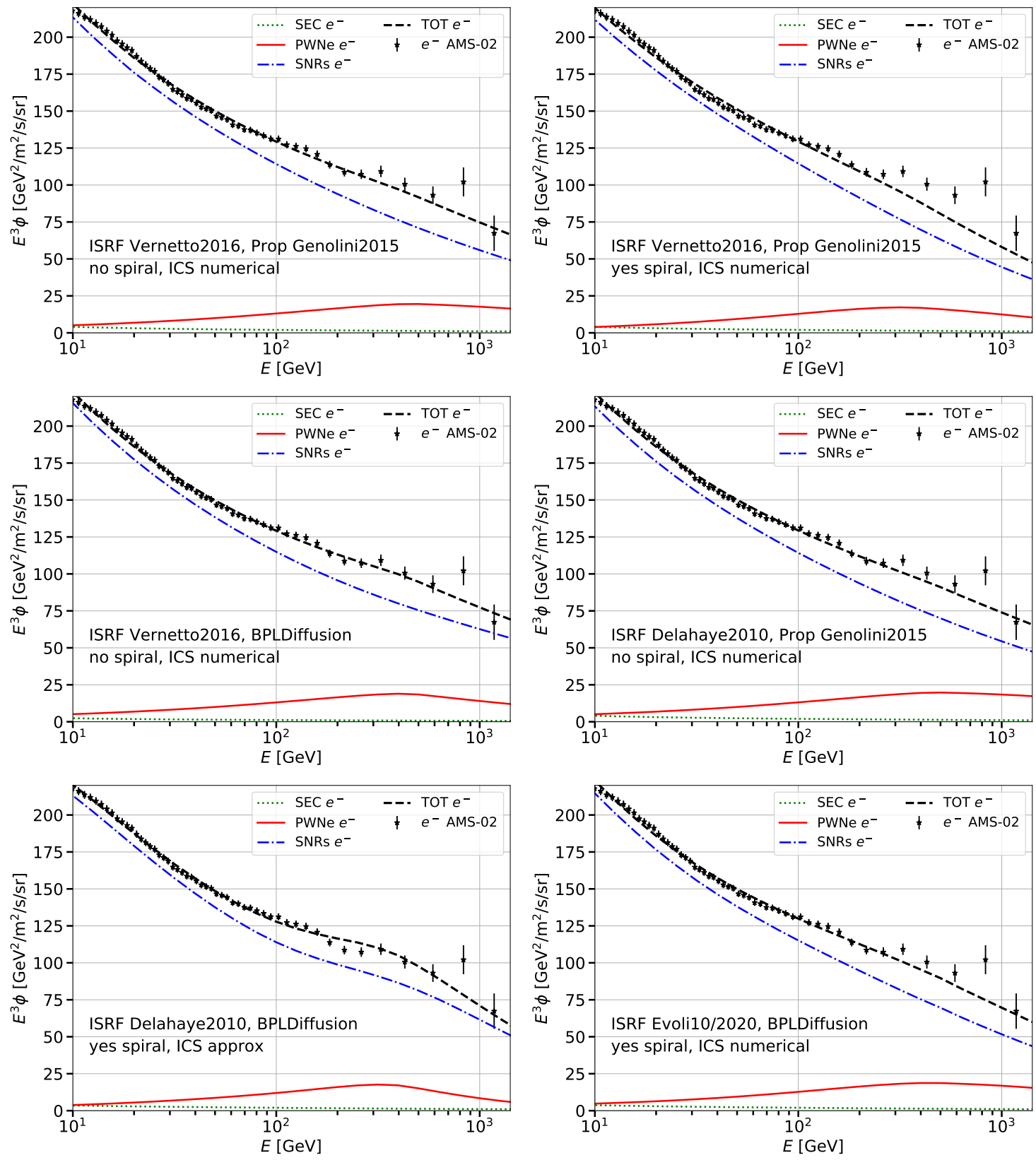

Figure 4: Flux of $e^{-}$from SNRs (blue dot-dashed line), PWNe (red solid line) and secondary production (green dotted line) as derived from a combined fit to the $e^{ \pm}$AMS-02 data. We also show the total contribution (black dashed line) and the AMS-02 data (black data points). Each plot refers to one of the first six cases reported in Tab. 1. 